\title{
Identification of RHOXF2 (PEPP2) as a cancer-promoting gene by expression cloning
}

\author{
FUMI SHIBATA-MINOSHIMA ${ }^{1,2^{*}}$, TOSHIHIKO OKI ${ }^{1,2^{*}}$, NORIKO DOKI ${ }^{1,2}$, FUMIO NAKAHARA $^{1,2}$, \\ SHUN-ICHIRO KAGEYAMA $^{3}$, JIRO KITAURA ${ }^{1,2}$, JUNYA FUKUOKA ${ }^{3}$ and TOSHIO KITAMURA ${ }^{1,2}$ \\ Divisions of ${ }^{1}$ Cellular Therapy and ${ }^{2}$ Stem Cell Signaling, the Institute of Medical Science, \\ the University of Tokyo, 4-6-1 Shirokanedai, Minatoku, Tokyo 108-8639; ${ }^{3}$ Department of \\ Surgical Pathology, Toyama University, 2630 Sugitani, Toyama 930-0194, Japan
}

Received June 27, 2011; Accepted July 28, 2011

DOI: $10.3892 /$ ijo.2011.1173

\begin{abstract}
Multiple mutations contribute to establish cancers. We have searched for potential oncogenes by screening cDNA libraries derived from gastric cancer cell lines, pancreatic cancer cell lines and glioma cell lines, using retrovirus-mediated expression cloning. Two types of interleukin-3 (IL-3)dependent cell lines, Ba/F3 and HF6, were transduced with the cDNA libraries and several genes that render these cells factor-independent were identified including PIM-1, PIM-2, $P I M-3, G A D D 45 B$ and reproductive homeobox genes on the $\mathrm{X}$ chromosome gene $\mathrm{F} 2$ (RHOXF2). Although no mutation in these genes was found, these molecules were highly expressed in cancer cell lines and they may play important roles in cell transformation. Among them, we focused on a transcriptional repressor RHOXF2. Transduction of RHOXF2 rendered HF6 cells factor-independent, while knockdown of RHOXF2 inhibited growth of the $\mathrm{HGC} 27$ gastric cancer cell line which highly expresses RHOXF2. In addition, RHOXF2-transduced HF6 cells quickly induced leukemia when transplanted into sublethally irradiated mice. Moreover, RHOXF2 is highly expressed in some leukemia cell lines and a variety of human cancer samples including colon and lung cancers. Thus, these results indicate that RHOXF2 is involved in carcinogenesis.
\end{abstract}

\section{Introduction}

Cancer develops through a multistep process involving a variety of gene alterations and epigenetic changes $(1,2)$. Genes that are commonly altered include $\mathrm{Rb}$, p16 ${ }^{\mathrm{INK} 4 \mathrm{a}}$, VHL, Ras, p53 and APC $(2,3)$. Fusion genes resulting from chromosomal translo-

Correspondence to: Dr Toshio Kitamura, Division of Cellular Therapy, the Institute of Medical Science, the University of Tokyo, 4-6-1 Shirokanedai, Minatoku, Tokyo 108-8639, Japan

E-mail: kitamura@ims.u-tokyo.ac.jp

${ }^{*}$ Contributed equally

Key words: c-DNA library screening, factor-independent growth, homeobox gene cations are established hallmarks of hematopoietic malignancies and include BCR-ABL, MLL-fusions and AML1-ETO (6). More recently, fusion genes have also been identified in solid tumors, for example EML4-ALK in lung cancer (4), EWS-FLI-1 in Ewing's sarcoma (5) and ETV6-NTRK3 in fibrosarcoma and breast cancer (6). Oncogenic mutations were originally identified using transfected NIH3T3 cells (7) in the focus-forming assay. A combination of the focus-forming assay with inoculation of NIH3T3 cells in vivo later increased the sensitivity to detect oncogenic potential (8). Recently, Soda et al identified a fusion gene EML4-ALK in non-small cell lung cancer by retrovirusmediated expression cloning using transformation of the NIH3T3 cells as an assay (4). EML4-ALK is now a promising molecular therapeutic target; ALK inhibitors proved to be effective in the therapy for non-small cell lung cancer patients bearing this fusion gene (9). Detection of oncogenes has largely depended on the classical focus-forming assay with NIH3T3, which mainly detects activated mutations of Ras and gene alterations that activate the Ras pathway (7). To detect different classes of oncogenic mutations, different assays are likely to be required.

In this study, two hematopoietic cell lines, Ba/F3 (10) and HF6 (11), were used to search for potential oncogenes in several cancer types. Both cell lines are interleukin-3 (IL-3)-dependent early myeloid cells, but to become factor-independent, $\mathrm{Ba} / \mathrm{F} 3$ requires JAK-STAT signaling and HF-6 requires Ras activation (11-13). Genes were tested for the ability to render these cell lines factor-independent. Using this strategy, several genes from cDNA libraries of gastric cancer cell lines, pancreatic cancer cell lines and glioma cell lines were identified including PIM-1, PIM-2, PIM-3, GAD45B and RHOXF2. Although mutations in these genes were not found, overexpression of PIM-1, PIM-2, PIM-3, GAD45B and RHOXF2 rendered HF6 cells factor-independent, confirming the validity of the functional screening. Of the genes identified, we have focused on a homeobox protein, reproductive homeobox genes on the $\mathrm{X}$ chromosome F2 (RHOXF2), also known as PEPP2. Homeobox proteins, share a DNA-binding homeodomain motif of 60 amino acids, and are transcription factors that regulate development. The Rhox genes belong to a recently discovered homeobox family whose members are clustered on the X chromosome (14-16). Whereas the human RHOX family consists of three members, RHOXF1, RHOXF2 and RHOXF2B, the murine 
Rhox family consists of $>30$ members (14-16). Rhox proteins are expressed in germ cells, embryonic cells and somatic cells of reproductive tissue, and play important roles in embryonic, postnatal and adult development, especially of the male and female reproductive systems. Recently, human and mouse Rhox protein expression in some cancer cells including breast and colon cancer was reported (16), and expression of a mouse Rhox family member, Rhox 5, was found in 50-65\% of cancer cells $(17,18)$. Interestingly, Rhox protein expression is regulated by epigenetic mechanisms, and the treatment with DNA methyltransferase inhibitors, such as decitabine, induces Rhox expression (16). These findings indicate that Rhox proteins are involved in carcinogenesis. In the present study, it was found that knockdown of RHOXF2 attenuated the growth of a gastric cancer cell line HGC27 and overexpression of RHOXF2 in HF6 cells rapidly induced leukemia in transplanted mice. These results support a role for RHOXF2 in cell transformation.

\section{Materials and methods}

Cells. Human gastric cancer cell lines (HGC27, GCIY, KATOIII, MKN45, OCUM-1, AGS, MKN1, MKN7, MKN45, MKN74, NUGC3, SNU719, TMK-1), human pancreatic cancer cell lines (Bx-PC-3, AsPC-1, capan1) and human glioma cell lines (U87MG, T98G, U251) were maintained in Dulbecco's modified Eagle's medium (DMEM) supplemented with $10 \%$ fetal calf serum (FCS). Ecotropic and amphotropic retrovirus packaging cell lines, PLAT-E and PLAT-A respectively, were maintained in DMEM supplemented with $10 \% \mathrm{FCS}, 1 \mu \mathrm{g} / \mathrm{ml}$ puromycin and $10 \mu \mathrm{g} / \mathrm{ml}$ blasticidin (19). A murine pro-B cell line $\mathrm{Ba} / \mathrm{F} 3$ was maintained in RPMI-1640 with $10 \%$ FCS and $1 \mathrm{ng} / \mathrm{ml}$ mouse IL-3 (mIL-3), and a murine myelomonocytic cell line HF6 was maintained in RPMI-1640 with $20 \%$ FCS and $10 \mathrm{ng} / \mathrm{ml} \mathrm{mIL-3} \mathrm{(11).}$

Plasmids and primers. The coding region of RHOXF2 was amplified from HGC27 cDNA by PCR using Phusion polymerase (Finnzymes, Oy, Finland), then subcloned into pMXs-puro or pMXs-IG vectors $(19,20)$ and the sequence was confirmed. Primers for RHOXF2 used in RT-PCR were as follows; (forward primer: cggaccagtgtagccagtat; reverse primer: tgacctcttcagtaagcgaca).

To generate shRNA vectors, first two oligonucleotides were designed using the siRNA Target Finder (Ambion Inc., Austin, USA) and were synthesized as follows; RHOXF2-1 (sense: ggca cagcagcaggagaaa, antisense: tttctctgctgctgtgcc) and RHOXF2-2 (sense: gagccaaatggaggagaca, antisense: tgtctcctccatttggctc). These oligonucleotides were annealed and ligated to the pReps vector, which was kindly provided by T. Hara (21). Control shLuc vector targeting firefly luciferase was constructed in the same way.

Generation of anti-RHOXF2 antibody. Anti-RHOXF2 polyclonal antibody was generated from the serum of rabbits immunized with the purified GST-RHOXF2 fusion protein (Scrum Inc., Tokyo, Japan).

Retroviral gene transduction. Retroviral transduction was performed as described $(19,20)$. Briefly, ecotropic and amphotropic retroviruses were generated by the packaging cell lines
Plat-E and Plat-A, respectively, and then transiently transfected with a pMXs-based construct using FuGENE 6 reagent (Roche Applied Science, Basel, Switzerland). Two days after transfection, ecotropic and amphotropic retroviruses were collected and used to infect mouse HF6 cells or human HGC27 cells, respectively.

Screening of cDNA libraries. pMXs-based cDNA libraries were generated from gastric cancer cell lines, pancreatic cancer cell lines and glioma cell lines, as previously described (13). Each cDNA library was retrovirally introduced into two IL-3dependent cell lines, Ba/F3 and HF6. Two days after transduction, the cells were seeded into 96-well plates in the absence of IL-3 in order to select factor-independent clones. To identify the cDNA that conferred $\mathrm{Ba} / \mathrm{F} 3$ or HF6 cells with factor-independency, the integrated cDNAs were isolated from factor-independent clones by genomic PCR and were sequenced using primers specific to the retroviral vector sequence.

Soft agar colony formation. One thousand cells were suspended in $0.6 \%$ agar supplemented with complete culture medium. This suspension was layered over $1.2 \%$ agar-medium bottom layer in $35-\mathrm{mm}$ dishes. After 14 days, colonies were counted.

Mouse bone marrow transplantation (BMT). One million HF6 cells transduced with empty (mock) or RHOXF2-containing retroviral vector were injected into the tail vein of sub-lethally irradiated (5.25 Gy) C57BL/6 (Ly-5.1) 8-week-old mice. Overall survival of the transplanted mice was analyzed using the Kaplan-Meier method. Animal studies were conducted in according to the guidelines of the Animal Care Committee at the Institute of Medical Science, University of Tokyo.

Generation of tissue microarray. Tissue microarrays (TMA) were generated from 1150 cases of 14 common cancer types as previously described (22). In addition, TMA composed of 280 non-neoplastic adjacent tissues from the same patients were made using identical methods. Each sample of TMA was cut in $4-\mu \mathrm{m}$ section for immunohistochemical analysis. Tissue microarray methods in the present study were approved by the Ethics Committee of Toyama University (no. 19-12).

Immunohistochemical analysis of tissue microarrays. Immunohistochemical detection of RHOXF2, and statistical analyses of results were carried out as previously described (22). In short, after deparafinization and rehydration, sections were processed for heat-induced antigen retrieval at $125^{\circ} \mathrm{C}$ and incubated with diluted antibody (1:500) for $30 \mathrm{~min}$ at room temperature. Antibody staining was visualized using the Envision ${ }^{+}$system (Dako, Kyoto, Japan) and diaminobenzidine.

Nuclear staining was scored according to 4 grades ( 0 , none; 1, mild; 2 , moderate; 3 , marked). Scores 0 and 1 were considered negative while scores 2 and 3 were considered positive. RHOXF2 staining of sections of an embedded pellet of HF6 cell line and colonic mucosa were used as positive and negative staining controls, respectively (data not shown).

\section{Results}

Expression cloning of potential oncogenes from cancer cell lines. cDNA was generated from gastric cancer cell lines, 
Table I. Characteristics of c-DNA libraries.

\begin{tabular}{lccc}
\hline & Gastric Cancer & Pancreatic cancer & Glioma \\
\hline Cell lines & HGC-27, OCUM-1, MKN45, GCIY, KATOIII & BxPC-3, AsPC-1, capan1 & U87MG, T98G, U251 \\
Library size & $4.5 \times 10^{6}$ & $3 \times 10^{6}$ & $3 \times 10^{6}$ \\
Avarage length & 1.2 & 1.4 & 1.3 \\
of cDNAs $(\mathrm{kb})$ & & & \\
\hline
\end{tabular}

The name of the cell lines used for making each library (cell lines), the numbers of independent cDNA clones of each library (library size), and the average length of the cDNAs included in each library (avarage length of cDNAs) are described.

Table II. Details of the isolated clones from screening. ${ }^{\text {a }}$

\begin{tabular}{|c|c|c|c|}
\hline & Gastric & Pancreatic & Glioma \\
\hline Total isolated clone & 5 & 2 & 6 \\
\hline The clones with full ORF & PIM-1,3, RNF67, RHOXF2 & PIM-1, GADD45B & PIM-2,3, CSF3, GADD45B \\
\hline The clones with partial ORF & TENC1, RAF1 & & FOXM1, ATXN1 \\
\hline The candidate oncogenes & PIM-1,3, RHOXF2 & PIM-1, GADD45B & PIM-2,3, GADD45B \\
\hline \multicolumn{4}{|c|}{$\begin{array}{l}\text { 'The clones isolated from } 3 \text { different c-DNA libraries are shown. The clones consist of two categories, the clones with full length of open } \\
\text { reading frame (ORF) of cDNAs (the clones with full ORF) and the clones with partial sequence of ORF (the clones with partial ORF). The } \\
\text { names of the genes which the clones of each category from } 3 \text { kinds of libraries contain, are shown in the table. Among the genes from the } \\
\text { clones with full length ORF, some genes rendered HF6 factor-independent and the names of the genes are indicated in the table (the candidate } \\
\text { oncogenes). }\end{array}$} \\
\hline
\end{tabular}

pancreatic cancer cell lines and glioma cell lines as described, and a cDNA library was constructed from the mixed cDNAs from each cancer in a retrovirus vector pMXs (20) (Table I). The resulting library was introduced into IL-3-dependent $\mathrm{Ba} /$ F3 cells and HF6 cells via retrovirus infection, and the infected cells were cultured in the absence of IL-3 to identify potential oncogenes that render the cells factor-independent. Several factor-independent clones were established from HF6 transfectants and PIM-1, PIM-2, PIM-3, GADD45B and RHOXF2 were isolated from these clones by genomic PCR (Table II). These genes were introduced into HF6 cells and the ability of these genes to confer HF6 cells with autonomous growth was confirmed (data not shown). However, no mutation was found in these genes, indicating that overexpression of these genes alone could transform HF6 cells.

Expression of RHOXF1 and F2 in a variety of cell lines. The Rhox family consists of 30 members in mice and 3 members in humans, RHOXF1, F2 and F2B. Interestingly, expression of RHOXF1 and F2 was almost mutually exclusive in gastric cancer cells examined (Fig. 1A). HGC27 (undifferentiated carcinoma), NUGC3 (poorly differentiated adenocarcinoma) and TMK-1 (poorly differentiated adenocarcinoma) cell lines express RHOXF2 but not RHOXF1. On the other hand, GCIY (scirrhous), MKN1 (adeno-squamous), MKN7 (well differentiated adenocarcinoma), MKN74 (moderately differentiated adenocarcinoma) and SNU719 (well differentiated adenocarcinoma) cell lines express RHOXF1 but not RHOXF2. These results suggested complementary roles for RHOXF1 and F2.

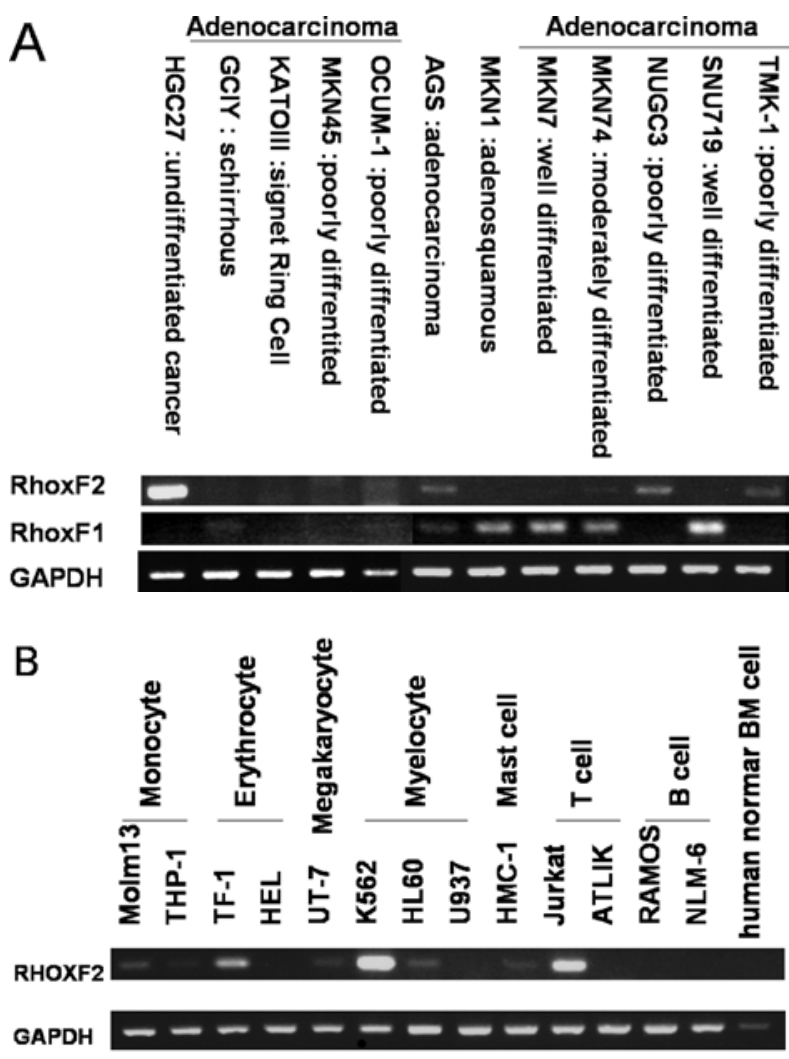

Figure 1. Expression of RHOXF2 in cancer cell lines. Expression of RHOXF2 was analyzed by RT-PCR. The expression in gastric cancer cell lines (A) and cell lines derived from hematological malignancies (B) are shown. 
A

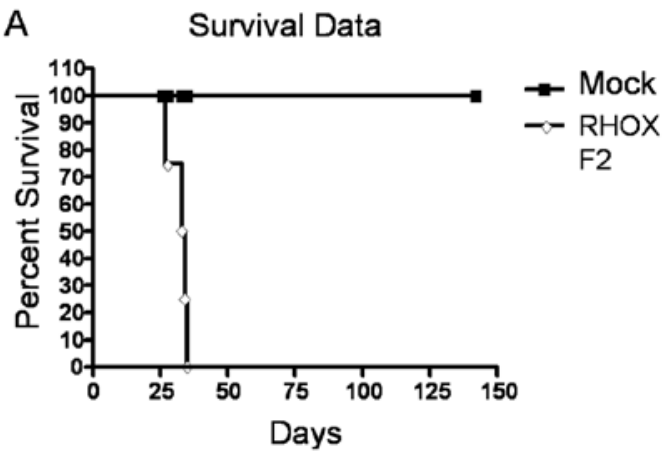

B

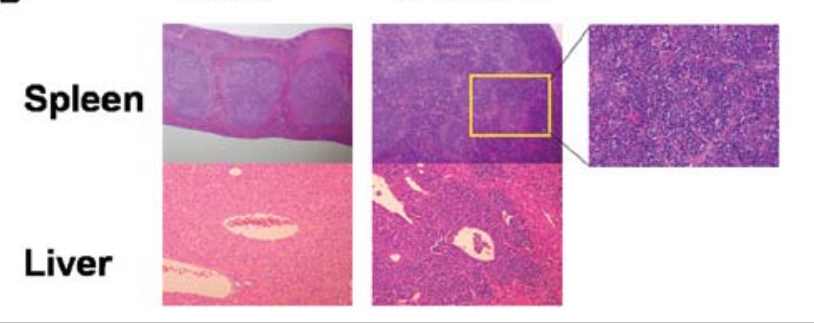

\begin{tabular}{lll}
\hline $\begin{array}{c}\text { Cytospin } \\
\text { of }\end{array}$ & BM & PB \\
RHOXF2 \\
BMT mice
\end{tabular}

Figure 2. Leukemia-like disease was induced by HF6 transduced with RHOXF2 but not by parental HF6 cells. The effects of RHOXF2 overexpression were analyzed using a mouse BMT model. Kaplan-Meier survival curves for mice transplanted with HF6-mock cells (black squares) or HF6-RHOXF2transfectants (white diamonds) are shown (A). Hematoxylin \& eosin-stained histological samples and May-Giemsa-stained cytospins revealed that hepatosplenomegaly caused by a leukemia-like cell infiltration was observed in mice transplanted with HF6-RHOXF2-trasnfectants (B).

The undifferentiated to poorly differentiated gastric cancers tended to express RHOXF2, while the adenocarcinoma cell line AGS appeared to express both RHOXF1 and F2.

RHOXF2 was also highly expressed in the human immature leukemic cell lines including TF-1 and K562 (Fig. 1B). It was also expressed in the other human hematopoietic cell lines, MOLM13, UT-7, HL60, MHC-1 and Jurkat (Fig. 1B).

Effects of overexpression of RHOXF2 in HF6 cells. HF6 cells that were manipulated to express RHOXF2 proliferated autonomously in vitro in the absence of IL-3 (data not shown). Next the effects of RHOXF2 overexpression in vivo were examined using a mouse BMT model. While unmanipulated HF6 cells did not induce disease in 5 months after the transplantation into sub-lethally irradiated mice, HF6 cells expressing RHOXF2 rapidly induced leukemia-like disease (Fig. 2A). In these mice, the leukemic cells infiltrated to the liver and spleen, causing hepato-splenomegaly (Fig. 2B).

Effects of RHOXF2 knockdown in HGC27. Next, the effects of RHOXF2 knockdown were evaluated. To achieve this, shRNA specific for RHOXF2 was retrovirally introduced into HGC27 gastric cancer cells which express RHOXF2 at high levels
A
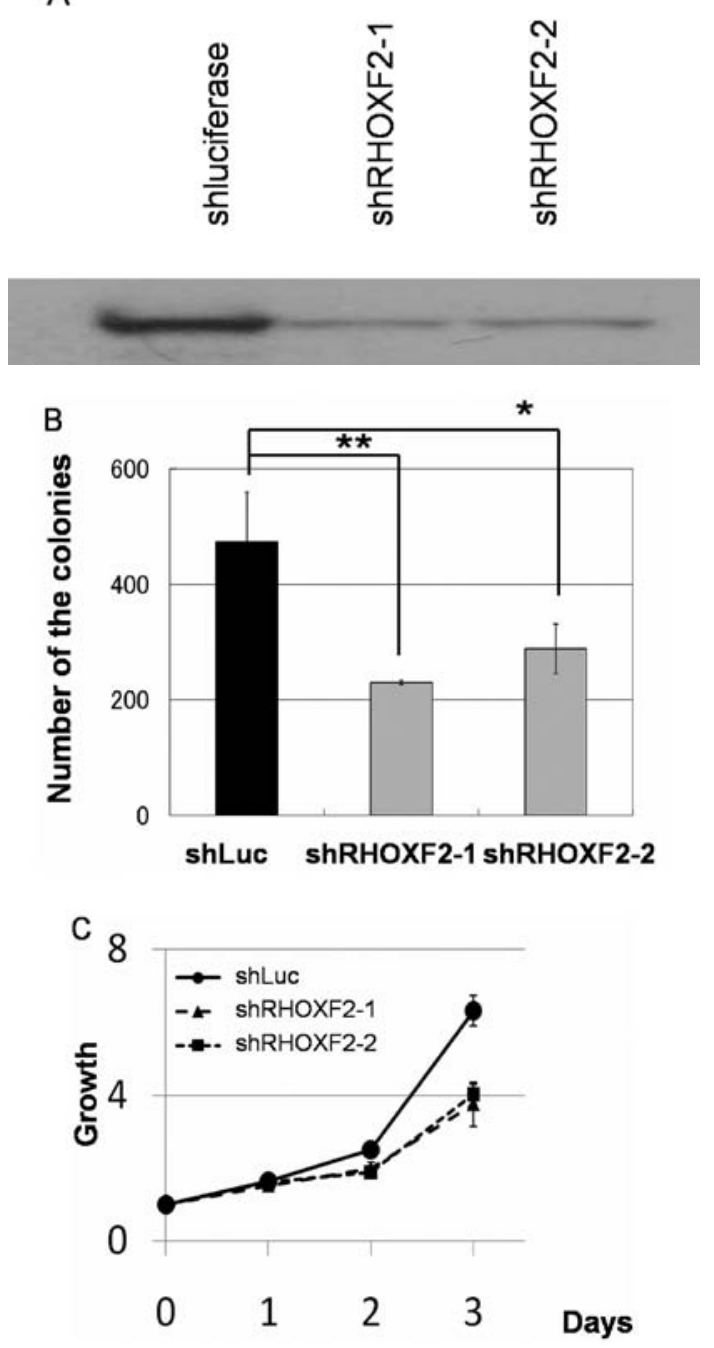

Figure 3. Knockdown of RHOXF2 attenuated the growth of a gastric cancer cell line, HGC27. Two shRNA vectors, shRHOXF2-1 and shRHOXF2-2, were designed to knockdown RHOXF2. Both shRNAs effectively inhibited the expression of RHOXF2 protein (A). shLuciferase vector was used as a control (shLuc). Colony formation in soft agar (B) and cell growth (C) of HGC27 transfectants were inhibited by shRHOXF2-1 (squares) and shRHOXF2-2 (triangles) but not by shLuc (circles). Statistical analysis was performed using the t-test; ${ }^{*} \mathrm{P}<0.05,{ }^{* *} \mathrm{P}<0.01$. All experiments were conducted in triplicates. The result shown here is a representative of 3 independent experiments.

(Fig. 3A). While the control shLuciferase did not inhibit the expression of RHOXF2, two RHOXF2 sh-RNA constructs efficiently suppressed the expression of RHOXF2 in HGC27 cells (Fig. 3A). Knockdown of RHOXF2 inhibited the colony forming ability of $\mathrm{HGC} 27$ cells in soft agar (Fig. 3B) and the growth rate in a liquid culture (Fig. 3C), implicating RHOXF2 in transformation and cell growth.

Expression of RHOXF 2 in a multi-cancerous tissue microarray. Expression of RHOXF2 in a variety of cancers and nonneoplastic tissues was evaluated using two TMA blocks (Fig. 4). RHOXF2 was not highly expressed in any of the normal tissues tested except for thyroid. RHOXF2 was highly expressed in some of the cancers including lung squamous cell carcinoma, thyroid tumors, colon, breast, gastric, prostate, ovarian and uterine corpus cancers. 


\section{A}

\section{Normal Tissue Microarray}
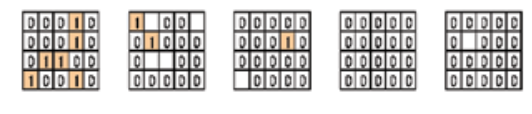

Intensity
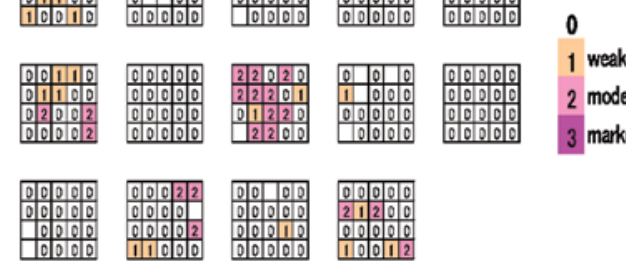

Lung Breast Bile tract Liver Stomach

Lung Kidney Thyroid Colon Prostate

Pancreas Bladder Ovrey Uterus

\section{B}

\section{Cancer tissue Microarray}
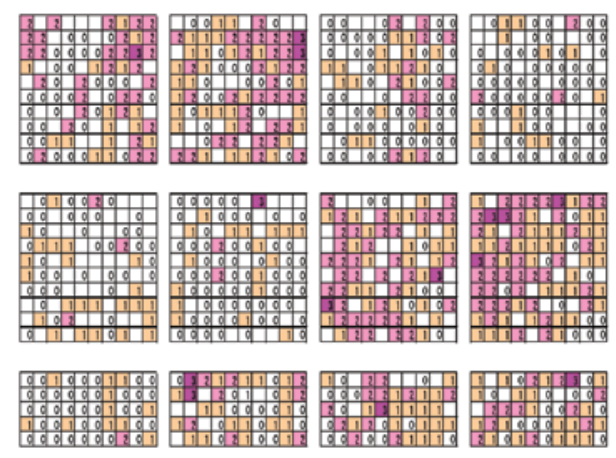

Lung(SCC) Breast

$\begin{array}{ll}\text { Lung(AD) } & \text { Kidney(RCC) } \\ \text { Pancreas } & \text { Bladder }\end{array}$

Bile tract

Thyroid

Ovary
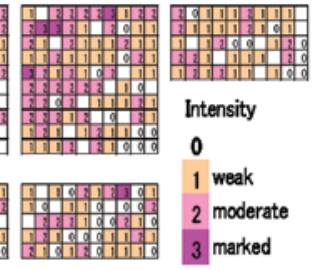

Intensity

0

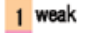

2 moderate

3 marked

Liver(HCC) Stomach

Colon

Uterus

Figure 4. Tissue microarray analysis. Immunohistochemical analysis of RHOXF2 expression in multi-tissue array is shown. (A and B) The array contains 280 non-neoplastic tissue samples and 1150 tumor tissue samples from 14 major types of cancers. Each column represents each case and the number in the column indicates the score corresponding to the level of RHOXF2 expression.

\section{Discussion}

In the present work, a transcriptional repressor RHOXF2 has been identified as a potential oncogene by functional expression cloning. Knockdown of RHOXF2 inhibited the growth and colony forming ability of the gastric cancer cell line HGC27. On the other hand, overexpression of RHOXF2 conferred IL-3-dependent HF6 cells with autonomous growth and leukemogenicity in vivo. In addition, RHOXF2 expression was found in a variety of cancer tissues. Recently, several studies have reported that expression of RHOXF2 is regulated by some epigenetic mechanisms, especially in cancer tissues (16). These findings suggest that RHOXF2 plays a role in tumorigenesis and that it can be controlled epigenetically. To elucidate the molecular mechanisms, further studies using gene chips and bioinformatics are required to identify the responsive genes downstream of RHOXF2. HF6 is a mouse bone marrowderived IL-3-dependent cell line immortalized by the fusion gene MLL-SEPT6, and activation of the Ras pathway in these cells can lead to transformation $(11,13)$. Ras activation frequently plays an important role in carcinogenesis and it is possible that genes repressed or enhanced by RHOXF2 are involved in this pathway.

It is now recognized that as in solid tumors, multiple mutations are involved in the development of hematopoietic malignancies. These mutations are classified into two groups; class I mutations include activating mutations of tyrosine kinases and oncogenes, and inactivating mutations of tumor suppressor genes such as p53, NF1, while class II mutations include dominant-negative mutations of transcription factors and chromatin modification enzymes (23-25). We have previously demonstrated that a class II mutation MLL-SEPT6 could immortalize mouse bone marrow cells by blocking the differentiation of hematopoietic progenitors (11-13). However, these immortalized cells still require interleukin 3 (IL-3) for their growth. Interestingly, class I mutations such as those that activate Ras signal or constitutively active receptors tyrosine kinase mutants, like FLT3-ITD, were able to fully transform the IL-3-dependent HF6 cells immortalized by class II mutations (13).

The assay system using NIH3T3 cells has been the gold standard for screening of oncogenes, however this system mainly detects activators of the Ras pathway (7). For this reason, different cell types have been needed to be tested in the search for the oncogenic mutations. In the present study, HF6 cells and Ba/F3 cells were used. HF6 cells and $\mathrm{Ba} / \mathrm{F} 3$ cells require activation of the Ras pathway and the JAK-STAT pathway, respectively, for the full transformation. In addition, we have established a cell line HF8 cells by introducing Hes1 to bone marrow progenitors. Neither Ras nor JAK/STAT activation could confer HF8 cells with autonomous growth (unpublished observation), indicating that these IL-3-dependent cell lines examined have distinct signaling profiles. The use of different cell lines could therefore, identify different classes of mutations. In the present study, PIM-1, PIM-2, PIM-3, GADD45B and RHOXF2 were shown to render HF6 cells factor-independent, demonstrating the potential for using cells of hematopoietic origin to identify oncogenic mutations. It is possible that different types of mutations and translocations could be identified, using distinct types of IL-3-dependent cell lines.

In conclusion, the transcriptional repressor RHOXF2 is expressed in a variety of cancers, and plays a critical role in tumorigenesis. The use of IL-3-dependent cell lines for screening cDNA libraries has potential as a strategy in the search for oncogenes.

\section{Acknowledgements}

We thank Dr T. Hara for kindly providing the retrovirus shRNA vector (pReps), and Mr. Masunori Kajikawa, ACTGen Inc., (Komagane, Japan) for providing cancer cell lines. This work was supported by grants from the Ministry of Health, Labor and Welfare.

\section{References}

1. Hanahan D and Weinberg RA: The hallmarks of cancers. Cell 100: 57-70, 2000.

2. Esteller M: Epigenetics in cancer. N Eng J Med 358: 1148-1159, 2008.

3. Jones PA and Baylin SB: The fundamental roles of epigenetic events in cancer. Nat Rev Genet 3: 415-428, 2002.

4. Soda M, Choi YL, Enomoto M, et al: Identification of the transforming EML4-ALK fusion gene in non-small-cell lung cancer. Nature 448: 561-566, 2007. 
5. Delattre O, Zucman J, Plougastel B, et al: Gene fusion with an ETS DNA-binding domain caused by chromosome translocation in human tumours. Nature 359: 162-169, 1992.

6. Mitelman F, Johansson B and Mertens F: Fusion genes and rearranged genes as a linear function of chromosome aberration in cancer. Nat Genet 36: 331-334, 2004.

7. Clark GL, Cox AD, Graham SM and Der CJ: Biological assays for Ras transformation. Method Enzymol 255: 395-412, 1995.

8. Takahashi K, Mitsui K and Yamanaka S: Role of ERas in promoting tumour-like properties in mouse embryonic stem cells. Nature 423: 541-545, 2003.

9. Gerber DE and Minna JD: ALK inhibition for non-small cell lung cancer: from discovery to therapy in record time. Cancer Cell 18: 548-551, 2010.

10. Palacios R and Steinmetz M: IL3-dependent mouse clones that express B-220 surface antigen, contain ig genes in germ-line configuration, and generate B lymphocytes in vivo. Cell 41: 727-734, 1985.

11. Ono R, Nakajima H, Ozaki K, et al: Dimerization of MLL fusion proteins and FLT3 activation synergize to induce multiplelineage leukemogenesis. J Clin Invest 115: 919-929, 2005.

12. Onishi M, Nosaka T, Misawa K, et al: Identification and characterization of a constitutively active STAT5 mutant that promotes cell proliferation. Mol Cell Biol 18: 3871-3879, 1998.

13. Watanabe-Okochi N, Oki T, Komeno Y, et al: Possible involvement of RasGRP4 in leukemogenesis. Int J Hematol 89: 470-481, 2009.

14. Maclean JA II, Chen MA, Wayne CM, et al: Rhox: a new homobox gene cluster. Cell 120: 369-382, 2005.

15. MacLean JA II and Wilkinson MF: The Rhox genes. Reproduction 140: $195-213,2010$
16. Li Q, Bartlett DL, Gorry MC, O'Malley ME and Guo ZS: Three epigenetic drugs up-regulate homeobox gene Rhox 5 in cancer cells through overlapping and distinct molecular mechanisms. Mol Pharmacol 76: 1072-1081, 2009.

17. Wilkinson MF, Kleeman J, Richards J and MacLeod CL: A novel oncofetal gene is expressed in a stage-specific manner in murine embryonic development. Dev Biol 141: 451-455, 1990.

18. Ono T, Sato S, Kimura N, et al: Serological analysis of BALB/C methylcholanthrene sarcoma Meth A by SEREX: identification of a cancer/testis antigen. Int J Cancer 88: 845-851, 2000.

19. Morita S, Kojima T and Kitamura T: Plat-E: an efficient and stable system for transient packaging of retroviruses. Gene Ther 7: 1063-1066, 2000.

20. Kitamura T, Koshino Y, Shibata F, et al: Retrovirus-mediated gene transfer and expression cloning: powerful tools in functional genomics. Exp Hematol 31: 1007-1014, 2003.

21. Fujino RS, Tanaka K, Morimatsu M, Tamura K, Kogo H and Hara T: Spermatogonial cell-mediated activation of an $\mathrm{I} \kappa \mathrm{B}$-independent nuclear factor- $\mathrm{\kappa B}$ pathway in sertoli cells induces transcription of the lipocalin-2 gene. Mol Endcrinol 20: 904-905, 2006.

22. Kitano H, Kageyama S, Hewitt SM, et al: Podoplanin expression in cancerous stroma induces lymphangiogenesis and predicts lymphatic spread and patient survival. Arch Pathol Lab Med 134: $1520-1527,2010$

23. Gilliland DG: Molecular genetics of human leukemias: new insights into therapy. Semin Hematoi 39: 6-11, 2002.

24. Dash A and Gilliland DG: Molecular genetics of acute myeloid leukaemia. Best Pract Res Clin Haematol 14: 49-64, 2001

25. Renneville A, Roumier C, Biggio V, et al: Cooperating gene mutations in acute myeloid leukemia: a review of the literature. Leukemia 22: 915-993, 2003. 